\title{
Mixed Time-scale Generalized Fair Scheduling for Amplify-and-Forward Relay Networks
}

\author{
Alireza Sharifian*, Petar Djukic*, Halim Yanikomeroglu*, Jietao Zhang ${ }^{\dagger}$ \\ *Department of Systems and Computer Engineering, Carleton University, Ottawa, Canada \\ e-mail: $\{$ alireza,djukic,halim\}@ sce.carleton.ca \\ ${ }^{\dagger}$ Wireless Research Department, Huawei Technologies Co., Ltd., Shenzhen, China \\ e-mail: jtzhang@huawei.com
}

\begin{abstract}
We devise an optimization framework for generalized proportional fairness (GPF) under different time scales for amplify-and-forward (AF) relay networks. In GPF scheduling, a single input parameter is used to change the fairness from throughput optimal, to proportionally fair and asymptotically to max-min fair. We extend the GPF scheduling to include a new input parameter, which determines the time-scale of fairness from short-term GPF to long-term GPF. We devise a low-complexity near-optimal algorithm to find schedules satisfying the given fairness criteria in a given time-scale. Simulations show that the proposed algorithm indeed allows the flexibility to change the fairness and its time-scale. To the best of our knowledge, this paper is the first to provide a multi-user scheduling framework for AF relays with both flexible fairness and flexible time-scales.
\end{abstract}

Index Terms-Long-term fairness, generalized fairness, amplify-and-forward relays.

\section{INTRODUCTION AND MOTIVATION}

We investigate combined short-term/long-term generalized fair resource scheduling for multi-user amplify-and-forward (AF) relays, which forward and multiplex data in orthogonal frequency division multiple access (OFDMA) networks. These relays are cost-effective, simpler to implement, and introduce less delay in comparison to other decode-and-forward relay based routers. As evident in today's wired networks, implementing hop-by-hop routing is a huge challenge at high data rates due to the hardware complexities of fast packet header inspection. AF relaying eliminates these issues from the very high-data rate wireless networks.

We devise a mixed time-scale generalized proportional fair (GPF) scheduling framework to make OFDMA-based AF relay scheduling fair to users. GPF scheduling is also known as $\gamma$-fair scheduling in the wired networking literature [1]. This framework is based on assigning utility functions to users, which take user's rate and a parameter $\gamma$ as inputs. Depending on the value of the $\gamma$ parameter, the rates that maximize the sum-utility gradually change resource allocation from throughput optimal to proportionally fair, and asymptotically to max-min fair.

To address the time-scale of fairness, our framework adds an additional parameter $\epsilon$ to $\gamma$-fair scheduling. For low values

This work was sponsored in part by Huawei Technologies Co., Ltd., Shenzhen, China. of $\epsilon$, the time-scale is long, so the scheduler provides longterm $\gamma$-fairness among the rates. On the other hand, for high values of $\epsilon$, the time-scale is short, so the scheduler provides short-term $\gamma$-fairness among the rates.

Previous works in AF relay networks found short-term fair user rates with optimization [2]-[5]. If the fairness is enforced in the constraints of the scheduling optimization [2], [3], [6], the minimum rates must be known in advance and the their feasibility must be checked by another mechanism. Another approach is to provide fairness with careful selection of the objective functions to achieve proportional fairness [4] or generalized fairness [5] similar to this paper. However, in those works fairness was limited to short-term time-scales. This paper extends the previous work to a more general framework where we can control the time-scale of the fairness.

We propose a gradient-based algorithm to quickly find schedules in each frame. Our framework and our algorithm consider AF frequency switching [7], which was not considered previously in fair multiuser cases [2], [3], [6], [8]-[10]. The algorithm allocates radio resources to users in iterations. In each iteration, rates are allocated to maximize the gradient of the sum-utility of user rates.

Our simulations show that the algorithm indeed allows the flexibility to change the fairness and its time-scale. The simulations also show that if the number of available resource blocks (RBs) in the frame is large, the fairness of the shortterm fair scheduling approaches the fairness of long-term one.

To the best of our knowledge, our work is the first to provide a scheduling framework for multi-user AF relays with both flexible rate fairness notion and flexible time-scales.

\section{System AND NeTWORK MODEL}

We consider OFDMA where orthogonal sub-carriers are grouped in time and frequency as RBs, with duration of $T_{b}$ seconds and a frequency span of $W_{b}$ Hertz. There are $N$ available sub-channels and $T$ RBs in each sub-channel in the frame to be assigned to $M$ users. The frame duration is $T_{c}=T T_{b}$ seconds. In the sequel, we consider a sector where a predetermined relay-station (RS) is serving the users. Our results can be easily extended to scenarios with multiple RSs.

The RS is an OFDMA-based AF relay, which multiplexes user data after receiving them from the BS. The relay amplifies the received symbols before multiplexing and retransmitting 
them on possibly different sub-channel. The received signal is sampled and processed with fast Fourier transform (FFT) to obtain the received modulation symbols, which are then stored in the RS's buffer. The RS may re-map the RBs from sub-channel to a different sub-channel, before performing the inverse FFT to obtain the output signal, not to couple a good sub-channel with a deep faded sub-channel. Multiple users may have RBs on the same sub-channel in the same frame.

The number of bits carried in an $\mathrm{RB}$ depends on the adaptive modulation and coding (AMC) used in the combined transmission over the two hops. We denote the number of bits in an RB, transmitted to user $m$ on sub-channel coupling $(i, j)$ in frame $k$ with $b_{i j}^{(m)}[k]$. The sub-channel coupling $(i, j)$ refers to the "coupling" of sub-channel $i$, used by the BS, with subchannel $j$, used by the RS. We use [.] to signify the discrete nature of time associated with OFDMA frames.

The rate of user $m$ in frame $k$ is determined from the number of RBs it is allocated in the frame $k$ and the AMC used in each RB:

$$
r_{m}[k]=\frac{1}{T_{c}} \sum_{i=1}^{N} \sum_{j=1}^{N} b_{i j}^{(m)}[k] x_{i j}^{(m)}[k],
$$

where $b_{i j}^{(m)}[k]$ is the number of bits that can be transmitted to user $m$ on sub-channel coupling $(i, j)$ in frame $k, x_{i j}^{(m)}[k]$ is the number of RBs assigned to user $m$ on sub-channel coupling $(i, j)$ in frame $k$, and $T_{c}$ is the frame duration in seconds. We use $x_{i j}^{(m)}[k]$ to indicate that the slot allocations in frame $k$ are the unknowns that the scheduling algorithm is searching for.

The slot allocations are integer number of RBs and are always allocated in pairs, since the AF relay cannot change the AMC of the received signals. Thus $T$ is an even number. This model is flexible enough to allow for many different frame structures. For example, if we limit allocation of a whole subchannels to one user at a time, $x_{i j}^{(m)}[k] \in\{0, T / 2\}$, otherwise a finer granularity is possible for $x_{i j}^{(m)}[k] \in\{0,1, \ldots, T / 2\}$.

The average rate is obtained from the instantaneous rates. At the beginning of frame $k+1$, after the scheduling algorithm finds RB allocation for frame $k$, the average rate of user $m$ is

$$
R_{m}[k]=\frac{1}{k} \sum_{j=1}^{k} r_{m}[k],
$$

so the relationship between average rate in frame $k$ and the average rate in frame $k-1$ is

$$
R_{m}[k]=\left(1-\frac{1}{k}\right) R_{m}[k-1]+\frac{1}{k} r_{m}[k] .
$$

In the sequel, we use a mixture of instantaneous and average rates to derive an optimization, which obtains fairness for different time-scales,

$$
\tilde{R}_{m}^{(\epsilon)}[k]=(1-\epsilon)\left(1-\frac{1}{k}\right) R_{m}[k-1]+\epsilon \frac{1}{k} r_{m}[k],
$$

where $1 / 2 \leq \epsilon \leq 1$ is a parameter determining, which of the two types of rates is more dominant. For $\epsilon=1 / 2$, the mixed rate simplifies to the scaled average rate

$$
\tilde{R}_{m}^{\left(\frac{1}{2}\right)}[k]=\frac{1}{2}\left[\left(1-\frac{1}{k}\right) R_{m}[k-1]+\frac{1}{k} r_{m}[k]\right]=\frac{1}{2} R_{m}[k],
$$

while for $\epsilon=1, \tilde{R}_{m}^{(1)}[k]=\frac{1}{k} r_{m}[k]$ is a scaled version of the instantaneous rate. We show shortly that the scaling factor does not change the results of the optimization framework. In the sequel, we call $\tilde{R}_{m}^{(\epsilon)}[k]$ the mixed time-scale rate for user $m$ in frame $k$.

A family of utility functions, which result in $\gamma$-fair [1] user rates is defined with

$$
U_{m}\left(\tilde{R}_{m}^{(\epsilon)}[k], \gamma\right)= \begin{cases}\frac{1}{1-\gamma}\left(\tilde{R}_{m}^{(\epsilon)}[k]\right)^{1-\gamma} & \text { if } \gamma \neq 1 \\ \log \left(\tilde{R}_{m}^{(\epsilon)}[k]\right) & \text { if } \gamma=1\end{cases}
$$

where $\gamma \geq 0$ is the parameter influencing the kind of fairness we expect and the term in the brackets is the mixed time-scale rate (4). The constant factor is necessary to make the utility concave for $\gamma>1$. In addition it is interesting to note that, with this kind of utility, the constant factor $\frac{1}{T_{c}}$ does not change the allocation. The allocation based on this utility is also independent of frequency span, which is hidden in $b_{i j}^{(m)}[k]$.

The sum utility over the user rates is the network utility

$$
U_{N}\left(\tilde{R}_{1}^{(\epsilon)}[k], \ldots, \tilde{R}_{m}^{(\epsilon)}[k], \gamma\right) \triangleq \sum_{m=1}^{M} U_{m}\left(\tilde{R}_{m}^{(\epsilon)}[k], \gamma\right) .
$$

User rates, which maximize the network utility for a specific $\gamma$ and $\epsilon$ are said to be $\gamma$-fair associated with time-scale of $\epsilon$.

Different rate fairness types can be achieved by changing the parameter $\gamma$, if the network utility function is used as the objective of an optimization [1]. For $\gamma=0$ maximizing the network utility corresponds to finding maximum averaged throughput, for $\gamma=1$, the averaged user rates maximizing the network utility are proportionally fair, and as $\gamma \rightarrow \infty$ the averaged rates maximizing the network utility become maxmin fair.

In the following section, we show how the $\epsilon$ and $\gamma$ parameters are used to design a flexible optimization framework, which finds rates satisfying different types of fairness and in different time-scales.

\section{Mixed Time-Scale $\gamma$-Fair AF Relay Scheduling}

Based on the $\gamma$-fair utility, we now formulate an optimization for $\mathrm{AF}$ relay that runs in each frame and finds instantaneous rates that maximize the network utility, which takes the mixed time-scale rates as inputs. Since different values of the $\epsilon$ parameter change the time-scale of the mixed time-scale rates, the same optimization can obtain short-term fairness, long-term fairness and a range between them. This framework unified the two separate optimizations, one may develop for short-term and long-term fairness.

In each frame $k$ we use the following optimization to maximize the network utility, (6), over all feasible instantaneous 
rates to find the $\gamma$-fair mixed time-scale rates

$$
\begin{aligned}
\max _{x_{i j}^{(m)}[k]} \sum_{m=1}^{M} \frac{\left((1-\epsilon)\left(1-\frac{1}{k}\right) R_{m}[k-1]+\epsilon \frac{1}{k} r_{m}[k]\right)^{1-\gamma}}{1-\gamma} \\
\text { s.t. } r_{m}[k]=\frac{1}{T_{c}} \sum_{i=1}^{N} \sum_{j=1}^{N} b_{i j}^{(m)}[k] x_{i j}^{(m)}[k], 1 \leq m \leq M, \quad(7 \mathrm{~b}) \\
R_{m}[k-1]=\left(1-\frac{1}{k}\right) R_{m}[k-2]+\frac{1}{k} r_{m}[k-1], \\
\quad \sum_{m=1}^{M} \sum_{j=1}^{N} x_{i j}^{(m)}[k] \leq \frac{T}{2}, 1 \leq i \leq N, \\
x_{i j}^{M}[k] \in\left\{\sum_{m=1}^{N} x_{i j}^{(m)}[k] \leq \frac{T}{2}, 1 \leq j \leq N,\right.
\end{aligned}
$$

where $M$ is the number of users, $N$ is the number of subchannels, $T_{c}$ is the frame duration, $b_{i j}^{(m)}[k]$ is the number of bits that can be transmitted to user $m$ on sub-channel coupling $(i, j)$ in the frame $k, x_{i j}^{(m)}[k]$ is the number of RBs assigned to user $m$ on sub-channel coupling $(i, j)$ in the frame $k, \gamma$ is the parameter that sets the type of fairness, and $\epsilon$ is the parameter that sets the time-scale of the fairness.

The objective function maximizes the network utility of mixed time-scale rates (6). Depending on the parameter $\gamma$, the optimization results in rates satisfying different rate fairness criteria. On the other hand, depending on the parameter $\epsilon$, the optimization results in different time-scales for the fairness, so it can obtain short-term or long-term fairness. Constraints (7b) relate the time allocations to user rates. Constraints (7d) and (7e) ensure that the total number of allocated RBs does not exceed what is available in the frame. Constraint (7f) ensures that the scheduling variables are integers.

The optimization variables, we are looking for, are $x_{i j}^{(m)}[k]$. All of $x_{i j}^{(m)}[l], l<k$ are fixed past decisions which reflect themselves in $R_{m}[k-1]$. Previous allocations make the current optimization biased.

We note that for $\epsilon=1 / 2$ the network utility objective simplifies to

$$
\begin{aligned}
U_{N}\left(\tilde{R}_{1}^{\left(\frac{1}{2}\right)}[k], \ldots, \tilde{R}_{m}^{\left(\frac{1}{2}\right)}[k], \gamma\right) \\
= \begin{cases}\left(\frac{1}{2}\right)^{(1-\gamma)} \frac{1}{1-\gamma}\left(R_{m}[k]\right)^{1-\gamma} & \text { if } \gamma \neq 1 \\
\log \left(\frac{1}{2}\right)+\log \left(R_{m}[k]\right) & \text { if } \gamma=1,\end{cases}
\end{aligned}
$$

so the constant factors, $\left(\frac{1}{2}\right)^{(1-\gamma)}, \log \left(\frac{1}{2}\right)$, can come out of the objective function and the optimization in terms of $\tilde{R}_{1}^{\left(\frac{1}{2}\right)}[k]$ is indeed equivalent to the optimization with $R_{m}[k]$ in the given $\gamma$-fairness.

If $\epsilon=1$, the optimization in each frame is independent of past allocations, dealing with the satisfaction only in frame $k$.

The optimization has a high computational complexity due

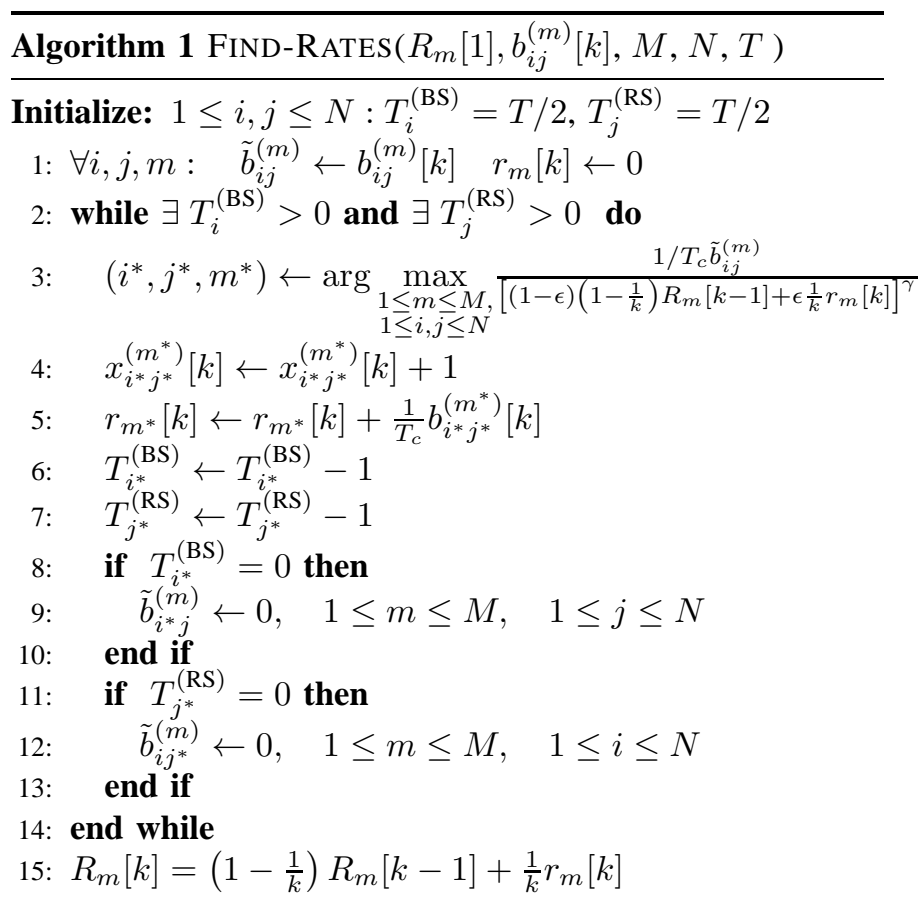

to non-linearity of the objective (7a) and constraint (7f), which ensures the integrality of time allocations. In addition the size of the optimization is very large. Therefore finding an algorithm with acceptable complexity is of interest. We devise a low-complexity sub-optimal algorithm to find mixed timescale rates, based on how the convex solver would find the best solution. The convex solver works in iterations. In each iteration, it finds the gradient of the objective function (in terms of time allocations) and increases the time allocations in the way that maximizes the gradient. Following this approach, our algorithm is based on the observation that if we can increase only one of $x_{i j}^{(m)}[k]$ in iteration, we should increase the time allocation of the sub-channel coupling $(i, j)$ for user $m$ with the highest partial derivative, to maximize the incremental change in the objective function.

Finding the sub-channel and user that maximize the partial derivative of the objective function,

$$
\begin{aligned}
& \frac{\partial}{\partial x_{i j}^{(m)}[k]} U_{N}\left(\tilde{R}_{1}^{(\epsilon)}[k], \ldots, \tilde{R}_{m}^{(\epsilon)}[k], \gamma\right) \\
& \quad=\frac{1}{T_{c}} \frac{b_{i j}^{(m)}[k]}{\left((1-\epsilon)\left(1-\frac{1}{k}\right) R_{m}[k-1]+\epsilon \frac{1}{k} r_{m}[k]\right)^{\gamma}},
\end{aligned}
$$

is the basis for the low-complexity sub-optimal algorithm (FIND-RATES). This can be also explained with Taylor expansion of the network utility.

Algorithm FIND-RATES works in iterations to find the mixed time-scale rates for frame $k$. Variables $T_{i}^{(B S)}$ and $T_{j}^{(R S)}$ keep track of the available slots on each sub-channel for the BS and the RS transmissions, respectively. The algorithm uses a copy of coupling rates $\tilde{b}_{i j}^{(m)}$ and make it zero when a sub-channel get exhausted. We use this copy because we need 
the original $b_{i j}^{(m)}[k]$ for updating the instantaneous rates. Since $\tilde{b}_{i j}^{(m)}$ is reset at each frame, there is no need for frame index regarding this variable. To make the algorithm run smoothly we initialize the average rates with a small value, $R_{m}[1]=\delta$. This approach is similar to [11], [12] where in the first step, when the rates are all zero, the best user is selected in terms of SNR.

In each iteration, the algorithm allocates time to the user and sub-channel that maximizes the partial derivative of the objective function. Step 3 finds the sub-channel coupling and user that maximizes the gradient decent of the objective function, according to (9). After finding the best sub-channel coupling, the allocation for that user is increased and the user's rate is updated in Step 4 and Step $5 . T_{i}^{(B S)}$ and $T_{j}^{(R S)}$ are then updated accordingly (Step 6 and Step 7). The copy of bits-per-slot values, $\tilde{b}_{i j}^{(m)}$, is also updated (set to zero) according to the availability of RBs, to ensure that allocated slots are not considered in the next iteration (Steps 8-13). Note that $b_{i j}^{(m)}[k] \mathrm{s}$ do not change as the algorithm runs and are used to find the user rates so far, on the other hand $\tilde{b}_{i j}^{(m)} \mathrm{s}$, change as the algorithm runs to find the best coupling for a selected user in each iteration. To avoid the complex search in Step 3, we implement the proposed algorithm with $M+1$ sorted lists containing SNRs of first-hop and the second-hops. Our sorting approach for exploiting the structure of Step 3 is similar to [7]. Due to space limitation we omitted the detail of implementation.

The complexity of the algorithm per frame is $\mathcal{O}((M+$ 1) $N \log (N)+M N \frac{T}{2}$ ) corresponding to our previous algorithm for short-term rate allocation [5].

For $\epsilon=1 / 2$, the algorithm is similar to the procedures proposed for single channel, single-hop, TDMA networks [13] and for conventional single-hop OFDMA networks [14]. The connection is not unexpected given the fact that both our approach and [13] use the same utility functions to achieve fairness. However, unlike those approaches, which find longterm fair rates, our algorithm can find either long-term or short-term fair rates. Also, note that, unlike [13], [14], our algorithm is for two-hop AF relay. For $\epsilon=1$, the algorithm is similar to the algorithm allocating short-term fair rates [5]. In this case there is no interdependency between frames. For $\gamma=0$, the algorithm is independent of $\epsilon$ and the algorithm reduces to a similar algorithm in [7] where no fairness is considered. Indeed, this non-obvious extension of [5] combines both approaches neatly and with a smooth transition.

\section{Simulation Results}

We consider a network of $M=15$ users in a sector connected to the BS through a RS in the sector. We randomly "drop" the users with a uniform density in the area around the relay. From the users' locations, we calculate each user's OFDM channel coefficients to the RS and from RS to BS using a detailed channel model [15] to find the number of bits carried in an RB on each sub-channel for 50 frames. Details of the simulation parameters are shown in Table I.
TABLE I

SIMULATION PARAMETERS

\begin{tabular}{|c|c|}
\hline Parameter & Value \\
\hline BS-RS Channel & Rician, $K=10 \mathrm{~dB}[15]$ \\
\hline BS-RS Shadowing & Log-normal, variance $3 \mathrm{~dB}$ \\
\hline BS-RS Doppler Shift & $4 \mathrm{~Hz}$ \\
\hline RS-Users Channel & Rayleigh ( [15]) \\
\hline RS-Users Shadowing & Log-normal, variance $5 \mathrm{~dB}$ \\
\hline RS-Users Doppler Shift & $37 \mathrm{~Hz}$ \\
\hline Path Loss & $38.4+2.35 \log 10(d) \mathrm{dB}$ \\
\hline Cell Radius & $1000 \mathrm{~m}$ \\
\hline BS-RS Distance & $500 \mathrm{~m}$ \\
\hline Transmit Power & $40 \mathrm{dBm}$ BS, $30 \mathrm{dBm}$ RS \\
\hline Antenna Gain & $10 \mathrm{~dB}$ BS, $5 \mathrm{~dB}$ RS, $0 \mathrm{~dB}$ Users \\
\hline Noise Figure & $2 \mathrm{~dB}$ RS, $2 \mathrm{~dB}$ Users \\
\hline
\end{tabular}

Jain's fairness index [16] of instantaneous rates for frame $k$ is given by

$$
\mathcal{J}[k]=\frac{\left(\sum_{m=1}^{M} r_{m}[k]\right)^{2}}{\left(M \sum_{m=1}^{M}\left(r_{m}[k]\right)^{2}\right)},
$$

which measures how similar the instantaneous rates are in frame $k$. To compare the impact of time dependent dynamic allocations on fairness of the rates, we use the Jain's index of the averaged rates

$$
\overline{\mathcal{J}}[k]=\frac{\left(\sum_{m=1}^{M} R_{m}[k]\right)^{2}}{\left(M \sum_{m=1}^{M}\left(R_{m}[k]\right)^{2}\right)},
$$

which shows how fair the averaged rates are up to frame $k$.

If the Jain's index is close to one, the rates are the most similar (flat), while for $\mathcal{J}[k]$ close to $\frac{1}{M}$, the rates are least similar so the system is in most unfair point.

Figure 1 shows the Jain's index of the averaged rates versus frame time index for different $\gamma$ and $\epsilon$. The number of available time slots is $T=8$ and the number of available sub-channels is $N=20$. We observe that as $\gamma$ is increased, the Jain's index is improved. This is expected since by increasing the $\gamma$, we are moving from throughput maximization to proportional fairness and asymptotically to max-min fairness. Figure 1 shows that Jain's index of long-term averaged rates for $\epsilon=1 / 2$ is greater than the Jain's index of long-term averaged rates for $\epsilon=1$. This is also expected due to the long-term fairness enabled by the scheduler for $\epsilon=1 / 2$. For $0.5<\epsilon<1$ the algorithm allows us to change the time scale of fairness. As $\epsilon \rightarrow 1$ the response of system in terms of fairness become smoother and we can avoid the overshoot and undershoot of the system response.

\section{CONClusion}

We devise an optimization framework for GPF in different time scales with frequency switching capability for AF relay networks. We extend GPF to include a new input parameter $\epsilon$, which determines the time-scale of fairness notion. For low values of $\epsilon$, the time-scale of target fairness notion is long, so 


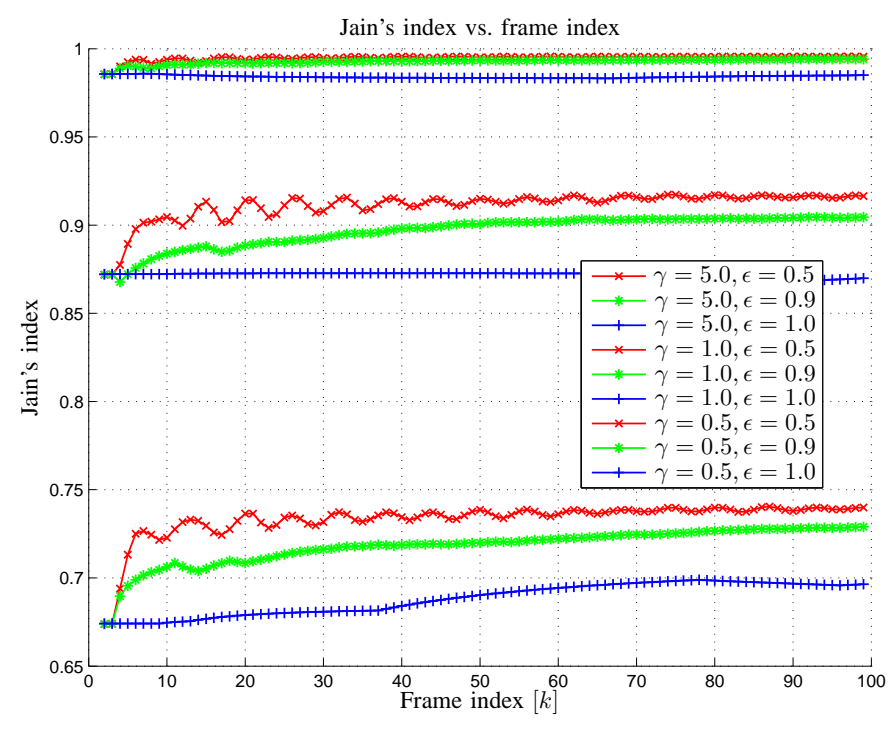

Fig. 1. Jain's index of the averaged rates vs. frame index.

the scheduler provides long-term $\gamma$-fairness among the rates. On the other hand, for high values of $\epsilon$, the time-scale is short, so the scheduler provides short-term $\gamma$-fairness among the rates. To the best of our knowledge, our work is the first to provide a scheduling framework for multi-user AF relays with both flexible fairness and flexible time-scales under which the fairness is met. We devise a low-complexity gradient-based algorithm to find schedules satisfying the given fairness notion in a given time-scale. Simulations show that the algorithm indeed allows the flexibility to change the fairness and its time-scale.

\section{REFERENCES}

[1] J. Mo and J. Walrand, "Fair end-to-end window-based congestion control," IEEE/ACM Transactions on Networking, vol. 8, no. 5, pp. 556567 , Oct. 2000

[2] M. Awad and X. Shen, "OFDMA-based two-hop cooperative relay network resources allocation," in IEEE International Conference on Communications (ICC), May 2008, pp. 4414-4418.

[3] X. Zhang, S. Chen, and W. Wang, "Multiuser radio resource allocation for multiservice transmission in OFDMA-based cooperative relay networks," EURASIP Journal on Wireless Communicattions and Networking, vol. 2009, pp. 1-13, 2009.

[4] W.-G. Ahn and H.-M. Kim, "Proportional fair scheduling in relay enhanced cellular OFDMA systems," in IEEE 19th International Symposium on Personal, Indoor and Mobile Radio Communications (PIMRC), Sept. 2008.

[5] A. Sharifian, P. Djukic, H. Yanikomeroglu, and J. Zhang, "Generalized proportionally fair scheduling for multi-user amplify-and-forward relay networks," in 71st IEEE Vehicular Technology Conference (VTC2010Spring), May 2010.

[6] G. Li and H. Liu, "Resource allocation for OFDMA relay networks with fairness constraints," IEEE Journal on Selected Areas in Communications, vol. 24, no. 11, pp. 2061-2069, Nov. 2006.

[7] A. Hottinen and T. Heikkinen, "Optimal subchannel assignment in a two-hop OFDM relay," in IEEE 8th Workshop on Signal Processing Advances in Wireless Communications (SPAWC), June 2007, pp. 1-5.

[8] J. Wang, Y. Zhao, and T. Korhonen, "Cross layer optimization with complete fairness constraints in OFDMA relay networks," in IEEE Global Telecommunications Conference (GLOBECOM), Dec. 2008.
[9] L. You, M. Song, J. Song, Q. Miao, and Y. Zhang, "Adaptive resource allocation in OFDMA relay-aided cooperative cellular networks," in 67th IEEE Vehicular Technology Conference, (VTC2008-Spring), May 2008, pp. 1925-1929.

[10] D. Zhang, Y. Wang, and J. Lu, "On QoS-guaranteed downlink cooperative OFDMA systems with amplify-and-forward relays: Optimal schedule and resource allocation," in IEEE Wireless Communications and Networking Conference (WCNC), April 2009.

[11] H. Rasouli and A. Anpalagan, "An asymptotically fair subcarrier allocation algorithm in OFDM systems," in IEEE 69th Vehicular Technology Conference (VTC Spring 2009), april 2009.

[12] W. Rhee and J. M. Cioffi, "Increase in capacity of multi-user OFDM system using dynamic sub-channel allocation," in 51st IEEE Vehicular Technology Conference (VTC2000-Spring), vol. 2, pp. 1085-1089.

[13] H. Kushner and P. Whiting, "Convergence of proportional-fair sharing algorithms under general conditions," IEEE Journal on Wireless Communication, vol. 3, no. 4, pp. 1250-1259, July 2004.

[14] G. Song and Y. Li, "Cross-layer optimization for OFDM wireless networks-part II: algorithm development," IEEE Transactions on Wireless Communications, vol. 4, no. 2, pp. 625-634, March 2005.

[15] L. Hentilä, P. Kyästi, M. Köske, M. Narandžić, and M. Alatossava, "Matlab implementation of the WINNER phase II channel model ver1.1," http://projects.celtic-initiative.org/winner+/phase_2_model.html, December 2007

[16] R. Jain, D. Chiu, and W. Hawe, "A quantitative measure of fairness and discrimination for resource allocation in shared computer system," in DEC Technical Report 301, 1984. 